\title{
Pedicle screw anchorage of carbon fiber-reinforced PEEK screws under cyclic loading
}

\author{
Richard A. Lindtner $^{1} \cdot$ Rene Schmid $^{1} \cdot$ Thomas Nydegger $^{2} \cdot$ Marko Konschake $^{3} \cdot$ Werner Schmoelz $^{1} \mathbb{C}$
}

Received: 18 August 2017 / Revised: 21 February 2018 / Accepted: 25 February 2018 / Published online: 1 March 2018

(c) The Author(s) 2018. This article is an open access publication

\begin{abstract}
Purpose Pedicle screw loosening is a common and significant complication after posterior spinal instrumentation, particularly in osteoporosis. Radiolucent carbon fiber-reinforced polyetheretherketone (CF/PEEK) pedicle screws have been developed recently to overcome drawbacks of conventional metallic screws, such as metal-induced imaging artifacts and interference with postoperative radiotherapy. Beyond radiolucency, CF/PEEK may also be advantageous over standard titanium in terms of pedicle screw loosening due to its unique material properties. However, screw anchorage and loosening of CF/PEEK pedicle screws have not been evaluated yet. The aim of this biomechanical study therefore was to evaluate whether the use of this alternative nonmetallic pedicle screw material affects screw loosening. The hypotheses tested were that (1) nonmetallic CF/PEEK pedicle screws resist an equal or higher number of load cycles until loosening than standard titanium screws and that (2) PMMA cement augmentation further increases the number of load cycles until loosening of CF/PEEK screws.

Methods In the first part of the study, left and right pedicles of ten cadaveric lumbar vertebrae (BMD $70.8 \mathrm{mg} / \mathrm{cm}^{3} \pm 14.5$ ) were randomly instrumented with either CF/PEEK or standard titanium pedicle screws. In the second part, left and right pedicles of ten vertebrae (BMD $56.3 \mathrm{mg} / \mathrm{cm}^{3} \pm 15.8$ ) were randomly instrumented with either PMMA-augmented or nonaugmented CF/PEEK pedicle screws. Each pedicle screw was subjected to cyclic cranio-caudal loading (initial load ranging from $-50 \mathrm{~N}$ to $+50 \mathrm{~N}$ ) with stepwise increasing compressive loads ( $5 \mathrm{~N}$ every 100 cycles) until loosening or a maximum of 10,000 cycles. Angular screw motion ("screw toggling") within the vertebra was measured with a 3D motion analysis system every 100 cycles and by stress fluoroscopy every 500 cycles.

Results The nonmetallic CF/PEEK pedicle screws resisted a similar number of load cycles until loosening as the contralateral standard titanium screws ( $3701 \pm 1228$ vs. $3751 \pm 1614$ load cycles, $p=0.89)$. PMMA cement augmentation of CF/PEEK pedicle screws furthermore significantly increased the mean number of load cycles until loosening by 1.63 -fold ( $5100 \pm 1933$ in augmented vs. $3130 \pm 2132$ in nonaugmented CF/PEEK screws, $p=0.015)$. In addition, angular screw motion assessed by stress fluoroscopy was significantly smaller in augmented than in nonaugmented CF/PEEK screws before as well as after failure.

Conclusions Using nonmetallic CF/PEEK instead of standard titanium as pedicle screw material did not affect screw loosening in the chosen test setup, whereas cement augmentation enhanced screw anchorage of CF/PEEK screws. While comparable
\end{abstract}

Electronic supplementary material The online version of this article (https://doi.org/10.1007/s00586-018-5538-8) contains supplementary material, which is available to authorized users.

Werner Schmoelz

werner.schmoelz@i-med.ac.at

1 Department of Trauma Surgery, Medical University of Innsbruck, Anichstraße 35, 6020 Innsbruck, Austria

2 icotec ag, Industriestraße 12, 9450 Altstätten, Switzerland

3 Division of Clinical and Functional Anatomy, Department of Anatomy, Histology and Embryology, Medical University of Innsbruck, Müllerstraße 59, 6020 Innsbruck, Austria 
to titanium screws in terms of screw loosening, radiolucent $\mathrm{CF} / \mathrm{PEEK}$ pedicle screws offer the significant advantage of not interfering with postoperative imaging and radiotherapy.

Graphical abstract These slides can be retrieved under Electronic Supplementary Material.
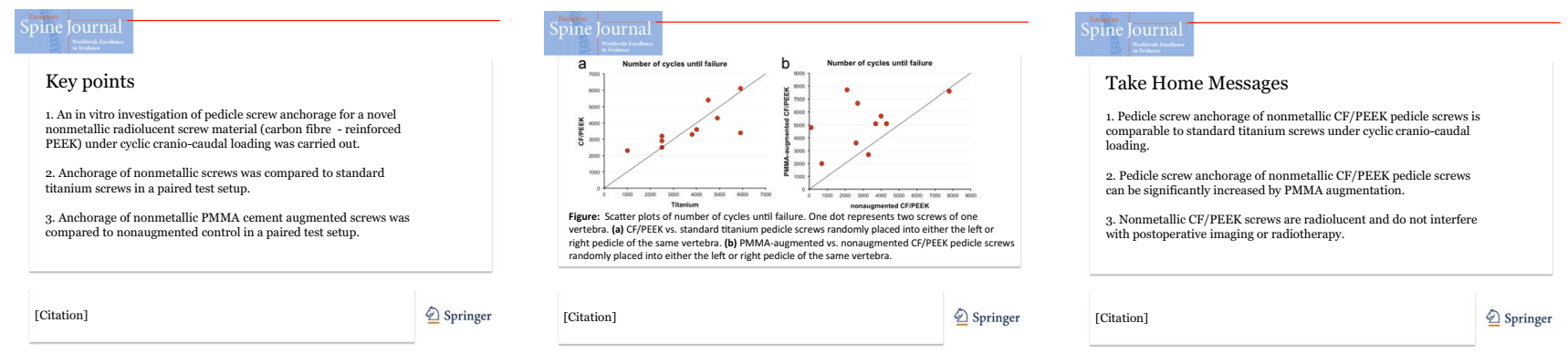

Keywords Pedicle screw $\cdot$ Pedicle screw loosening $\cdot$ Polyetheretherketone $\cdot$ PEEK $\cdot$ Carbon fiber-reinforced PEEK $\cdot$ Pedicle screw augmentation $\cdot$ Cyclic loading $\cdot$ Biomechanics $\cdot$ Osteoporosis $\cdot$ Elastic modulus $\cdot$ Spine $\cdot$ Posterior instrumentation . Spinal tumors

\section{Introduction}

Metal-induced artifacts significantly impede postoperative imaging of the spine $[1,2]$. To overcome this shortcoming of conventional metallic pedicle screws, nonmetallic highstrength carbon fiber-reinforced polyetheretherketone $(\mathrm{CF} /$ PEEK) pedicle screws have been developed. CF/PEEK is radiolucent and nonmagnetizable, and thus allows to minimize imaging artifacts in CT and MRI imaging [3-5]. This feature greatly facilitates postoperative radiological assessment of fusion and adjacent segment disease, postoperative evaluation of neural structures in case of newly developing neurological symptoms as well as radiological follow-up of spinal tumors after spinal stabilization. Moreover, radiolucency of CF/PEEK improves dose calculation for postoperative radiotherapy of spinal tumors and minimizes radiation scattering and tumor shielding caused by metallic implants [5, 6]. Beyond radiolucency, CF/PEEK may also be advantageous over standard titanium in terms of pedicle screw loosening due to its unique material properties. However, to the authors' best knowledge, screw anchorage and loosening of CF/PEEK screws-although critical prerequisites for successful clinical applicationhave not been biomechanically evaluated yet.

Pedicle screw loosening is one of the most common complications after posterior instrumentation of the thoracolumbar spine. Screw loosening rates of up to $15 \%$ have been reported for nonosteoporotic patients and even higher loosening rates of up to $60 \%$ have been observed in osteoporotic individuals [7]. Pedicle screw constructs are widely used to treat fractures, degenerative diseases, tumors and infection of the thoracolumbar spine as well as to correct spinal deformity [8]. Loosening of pedicle screws, however, may result in loss of reduction, spinal instability, painful nonunion after fusion and possibly even in recurrent pain despite successful fusion, and therefore may necessitate revision surgery [9-12]. There is an ongoing debate on how to improve pedicle screw anchorage and prevent screw loosening, and various strategies have been considered and evaluated. These strategies include various cement augmentation techniques, expandable screws and screw anchors, modifications of pedicle screw and screw thread design, alternative screw trajectories and screw insertion techniques, and perioperative teriparatide administration [13-15].

Until now, none of these strategies has been aimed at using an alternative, nonmetallic screw material, but all of them have rather still been based on and tailored to conventional metallic pedicle screws. Nonmetallic CF/PEEK, however, offers important advantages over titanium or stainless steel beyond radiolucency. CF/PEEK exhibits outstanding biomechanical properties with a modulus of elasticity closer to that of bone as compared to titanium and stainless steel [3]. CF/PEEK screw/rod constructs may therefore allow to better approximate the normal biomechanics of the spine via increased anterior column load-sharing, and consequently may promote interbody fusion and reduce the rate of adjacent segment disease [16-18]. Moreover, the lower modulus of elasticity theoretically allows to reduce stresses and micromotion at the bone-screw interface and thus to reduce pedicle screw loosening [18-20]. As to material properties, PEEK is a fully biocompatible, sterilizable and mechanically competent nonabsorbable semi-crystalline polymer [3]. Incorporation of reinforcing carbon fibers permits to tailor the elastic modulus and to substantially improve the 
mechanical properties of neat PEEK $[3,21]$. CF/PEEK, therefore, is much more like titanium in terms of material properties as compared to neat PEEK and may be a desirable compromise between stiff titanium and distinctly less rigid neat PEEK constructs to act as an adjunct to fusion. Both neat PEEK and CF/PEEK have already been successfully used for spinal applications, such as interbody cages, rods and interspinous implants [16, 22-24], whereas CF/PEEK pedicle screws have only recently become available.

The purpose of this biomechanical study therefore was (1) to compare screw loosening of nonmetallic CF/PEEK pedicle screws to standard titanium screws under cyclic loading and (2) to evaluate whether PMMA cement augmentation enhances screw anchorage of CF/PEEK pedicle screws. The two hypotheses tested were that (1) CF/PEEK pedicle screws resist an equal or higher number of load cycles until loosening than standard titanium screws and that (2) PMMA cement augmentation further increases the number of load cycles until loosening of CF/PEEK pedicle screws.

\section{Materials and methods}

\section{Specimen preparation}

Twenty fresh-frozen vertebrae (TH12-L5) from 8 donors [6 females and 2 males with a mean age of $76.1 \pm 12.2$ years (range 60-95 years)] were used for this study. The bodies were donated by people who had given their informed consent prior to death to use their bodies for scientific and educational purposes. The specimens were double shrinkwrapped and frozen at $-20{ }^{\circ} \mathrm{C}$ until testing. Quantitative computer tomography (GE Light-speed 16, GE Medical
Systems, Waukesha, WI, USA) including EFP (European Forearm Phantom calibration; QRM GmbH, Möhrendorf, Germany) was performed to assess bone mineral density (BMD) of each vertebra and to rule out structural abnormalities due to fracture as well as infectious, congenital or neoplastic disease. Mean BMD of vertebrae was $63.6 \pm 16.5 \mathrm{mg} /$ $\mathrm{cm}^{3}$ (range 37.1-92.4) and mean age of specimens was $73.5 \pm 11.3$ years. For biomechanical testing, specimens were thawed overnight at $6{ }^{\circ} \mathrm{C}$ with a consistent defrost period and then handled at room temperature. Soft tissues (including the intervertebral discs) were removed right before testing and all pedicle screws were implanted by an experienced spine surgeon (R. S.) in a standard fashion. After identification of the anatomical landmarks for screw placement, the entry point was determined and the pedicle was entered using a pedicle awl. Violation of the medial, lateral, cranial and caudal pedicle wall was ruled out by a ball-tipped probe and finally pedicle screws were inserted using a hand-driver. Baseline axial and lateral fluoroscopic images were obtained prior to testing to ensure correct pedicle screw position and to document cement distribution of augmented screws. The superior and inferior endplate of each individual vertebra was potted in PMMA (Technovit 3040, Heraeus Kulzer GmbH, Wehrheim, Germany) to allow for fixation of the specimens in the material testing machine.

\section{Pedicle screws and cement augmentation}

Two types of polyaxial pedicle screws of identical design (i.e., identical screw dimension, geometry as well as identical thread design) but manufactured from different screw materials were evaluated (Fig. 1a):

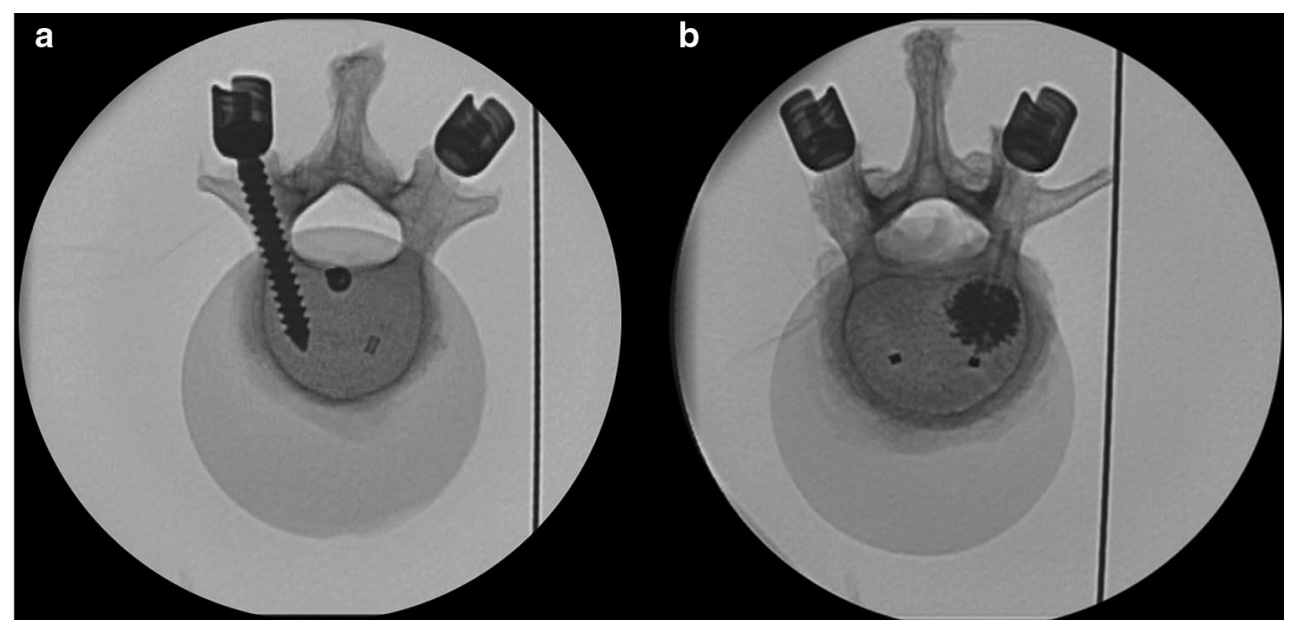

Fig. 1 Axial fluoroscopic images of instrumented vertebrae. a For comparison of CF/PEEK and standard titanium pedicle screws, left and right pedicles of each cadaveric vertebra were randomly instrumented with either a CF/PEEK (right) or a titanium control screw (left); b for comparison of PMMA-augmented (right) and nonaugmented CF/PEEK (left) pedicle screws, both pedicles of each vertebra were instrumented with $\mathrm{CF} / \mathrm{PEEK}$ pedicle screws and one screw was randomly selected for in situ augmentation with 2 ml PMMA cement 
1. $6.5 \mathrm{~mm} \times 45 \mathrm{~mm}$ carbon fiber-reinforced polyetheretherketone (CF/PEEK) pedicle screws (VADER ${ }^{\circledR}$ Pedicle System Carbon/PEEK, icotec ag, Altstätten, Switzerland) made from radiolucent and nonmagnetizable BlackArmor ${ }^{\circledR}$ Carbon/PEEK material. BlackArmor ${ }^{\circledR}$ is a combination of continuous, high-strength carbon fibers in a PEEK polymer matrix and a composite flow moulding process resulting in an interwoven 3D fiber architecture that provides improved strength and endurance properties. The screws are cannulated, fenestrated, and titanium coated in the area of the pedicle for improved osseointegration. A Tantalum marker is incorporated into the tip of the screw to enable visualization of the implant position in all state-of-the-art imaging modes (MRI, CT and X-ray).

2. $6.5 \mathrm{~mm} \times 45 \mathrm{~mm}$ standard titanium (Ti6Al4V ELI) pedicle screws (icotec Pedicle System Titanium, icotec ag, Altstätten, Switzerland) served as controls.

Cement augmentation of CF/PEEK pedicle screws was performed in situ by injecting 2 ml PMMA cement (Vertecem ${ }^{\mathrm{TM}} \mathrm{V}+$, DePuy Synthes, Oberdorf, Switzerland) through the cannulated and fenestrated screws.

\section{Test groups}

For comparison of CF/PEEK vs. standard titanium pedicle screws, left and right pedicles of ten isolated human lumbar vertebrae $[2 \times \mathrm{L} 1,2 \times \mathrm{L} 3,3 \times \mathrm{L} 4$ and $3 \times \mathrm{L} 5$; BMD $70.8 \pm 14.5 \mathrm{mg} / \mathrm{cm}^{3}$ (range $48.1-92.4$ )] obtained from 3 donors [ 2 females and 1 male; age: 60, 75 and 72 years $(69.0 \pm 7.9$ years)] were randomly instrumented with either $\mathrm{CF} / \mathrm{PEEK}$ or standard titanium pedicle screws of identical size and design (Fig. 1a).

For comparison of PMMA-augmented vs. nonaugmented $\mathrm{CF} / \mathrm{PEEK}$ pedicle screws, left and right pedicles of ten vertebrae $[1 \times \mathrm{TH} 12,1 \times \mathrm{L} 1,2 \times \mathrm{L} 2,1 \times \mathrm{L} 3,2 \times \mathrm{L} 4$ and $3 \times \mathrm{L} 5$; BMD $56.3 \pm 15.8 \mathrm{mg} / \mathrm{cm}^{3}$ (range 37.1-82.0)] from 5 donors [4 females and 1 male; age: 66, 70, 79, 92 and 95 years $(80.4 \pm 12.9$ years) $]$ were randomly instrumented with either PMMA-augmented or nonaugmented CF/PEEK pedicle screws (Fig. 1b).

\section{Cyclic loading}

Each pedicle screw was subjected to cyclic loading in cranio-caudal direction with stepwise increasing loads until total screw failure or a maximum of 10,000 load cycles using a servohydraulic biaxial material testing machine (858 Mini Bionix II, MTS, Eden Prairie, MN, USA). The instrumented vertebra was fixed to an $x-y$ plane bearing table in the testing machine allowing translation in the anterior-posterior as well as the medial-lateral plane during cyclic loading (Fig. 2). Each pedicle screw was attached to the actuator of the servohydraulic testing machine via a straight $\mathrm{CF} / \mathrm{PEEK}$ $\operatorname{rod}\left(\varnothing 5.5 \mathrm{~mm} \times 100 \mathrm{~mm}\right.$; VADER ${ }^{\circledR}$ Pedicle System, Carbon/PEEK, icotec ag, Altstätten, Switzerland). The loading $\mathrm{CF} / \mathrm{PEEK}$ rod was connected to the pedicle screw with a lever arm of $15 \mathrm{~mm}$ to the rotational axis to approximate the rotational axis of an instrumented spine and to also combine
Fig. 2 Biomechanical test setup. The instrumented vertebra was fixed to an $x-y$ plane bearing table and the tested screw was attached to the actuator (A) of the testing machine via a rod with a lever arm of $15 \mathrm{~mm}$ to the rotational axis (blue bar). A 3D motion analysis system (indicated by asterisks) was mounted to the screw head as well as the $x-y$ plane bearing table to measure angular screw motion within the fixed vertebra every 100 cycles. Additionally, stress fluoroscopy was performed every 500 cycles using a C-arm (X-ray tube visible in background). The red arrow indicates cyclic cranio-caudal loading. The green arrows indicate the degrees of freedom of the test setup during cyclic loading

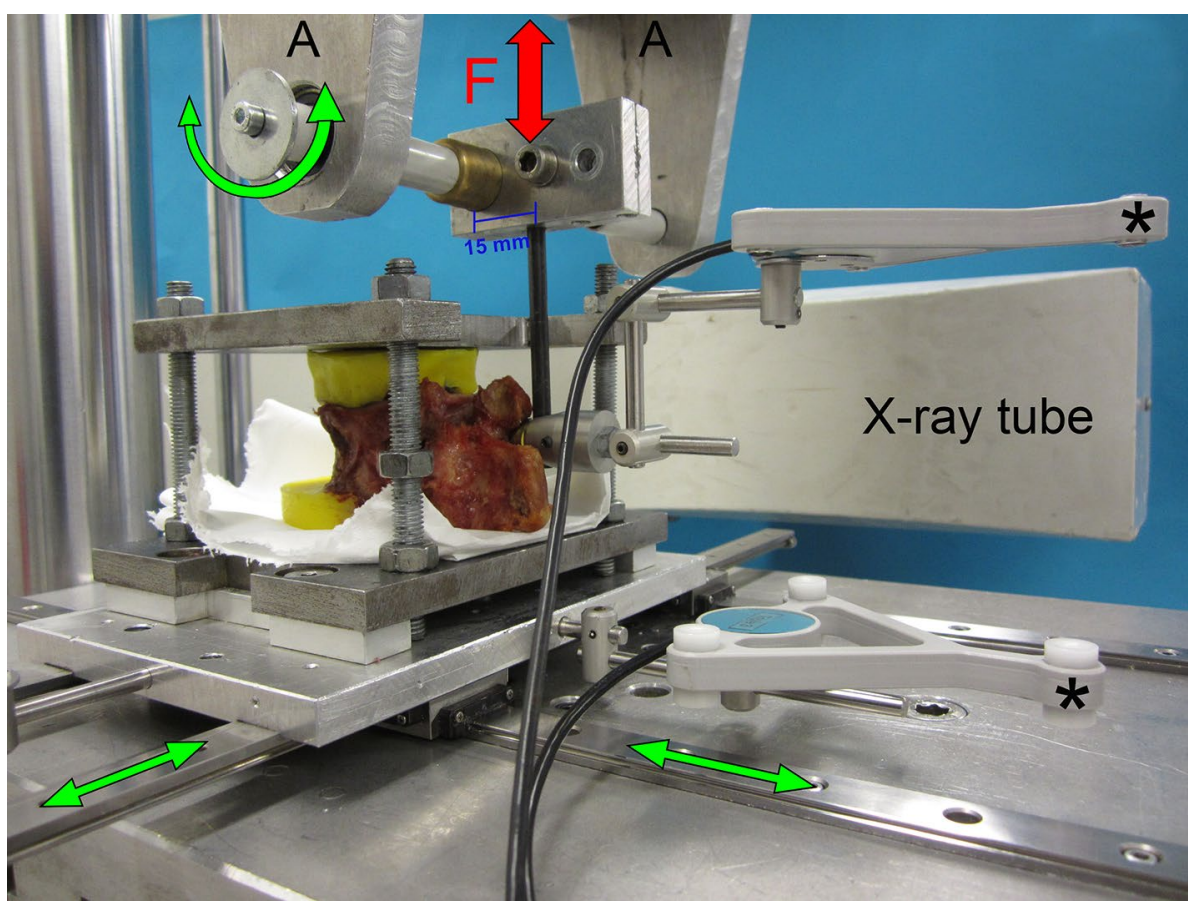


the applied axial loading with a bending moment on the pedicle screw $[25,26]$. Each pedicle screw was cyclically loaded with an initial load ranging from $-50 \mathrm{~N}$ (tension) to $+50 \mathrm{~N}$ (compression). The compression load was increased by $5 \mathrm{~N}$ every 100 cycles. The material testing machine was programmed in displacement control $(5 \mathrm{~mm} / \mathrm{s})$ with force limits. Cyclic loading was terminated after either 10,000 cycles or total failure of the pedicle screw. The latter was defined as $10 \mathrm{~mm}$ axial displacement of the actuator in caudal direction compared to the neutral unloaded stage.

A 3D motion analysis system (Zebris, Winbiomechanics, Isny, Germany) was mounted to the screw head and the $x-y$ plane bearing table to measure the relative angular screw motion (also known as "screw toggling" or "windshieldwiper motion") within the fixed vertebra every 100 cycles. In addition, lateral stress fluoroscopic images were obtained manually every 500 cycles at minimal [ $-50 \mathrm{~N}$ (tension)] and maximal (respective compression load limit) load (Fig. 3). Angular screw motion was then visualized by digitally superimposing both X-ray images obtained.

Upon completion of cyclic loading, a post-test data analysis was performed to evaluate in detail when pedicle screw loosening had occurred. Pedicle screws were considered loose if the overall angular screw motion exceeded $8^{\circ}$ compared to the neutral unloaded stage or if angular screw motion increased more than $1^{\circ}$ within 100 load cycles $[25$, 26].

\section{Statistical analysis}

All statistical analyses were performed using IBM $^{\circledR}$ SPSS Statistics ${ }^{\circledR}$ version 24 (IBM, Armonk, NY, USA). Metric scaled data are reported as arithmetic mean \pm standard deviation. A paired samples $t$ test was applied for comparisons between CF/PEEK and standard titanium pedicle screws as well as for comparisons between PMMA-augmented and nonaugmented CF/PEEK pedicle screws. Spearman's correlation was used to examine the relationship between number of load cycles until pedicle screw loosening and BMD. Statistical significance was defined as $p<0.05$.

\section{Results}

\section{CF/PEEK vs. standard titanium pedicle screws}

For comparison of CF/PEEK vs. standard titanium pedicle screws, the two types of pedicle screws were randomly placed into either the left or right pedicle of each of ten isolated human lumbar vertebrae [BMD $70.8 \pm 14.5 \mathrm{mg} / \mathrm{cm}^{3}$ (range 48.1-92.4)]. All screws loosened within the 10,000 load cycles.

The nonmetallic CF/PEEK pedicle screws resisted a similar number of load cycles until loosening as the contralateral titanium screws [3701 \pm 1228 (corresponding to $235 \pm 61 \mathrm{~N}$ ) vs. $3751 \pm 1614$ load cycles (corresponding to $239 \pm 80 \mathrm{~N}$ ), $p=0.89]$. CF/PEEK screws reached a higher number of load cycles until loosening in five specimens, exactly the same number of load cycles in one specimen and a lower number of load cycles in four specimens as compared to the contralateral titanium screws (Fig. 4). The correlation between number of load cycles until loosening and BMD was weak for both CF/PEEK and titanium screws $[r=0.33, p=0.35$ and $0.39, p=0.26$, respectively (Spearman correlation)].

Similarly, angular screw motion assessed by stress fluoroscopy at 1000 cycles, last measurement before and first measurement after failure did not significantly differ between

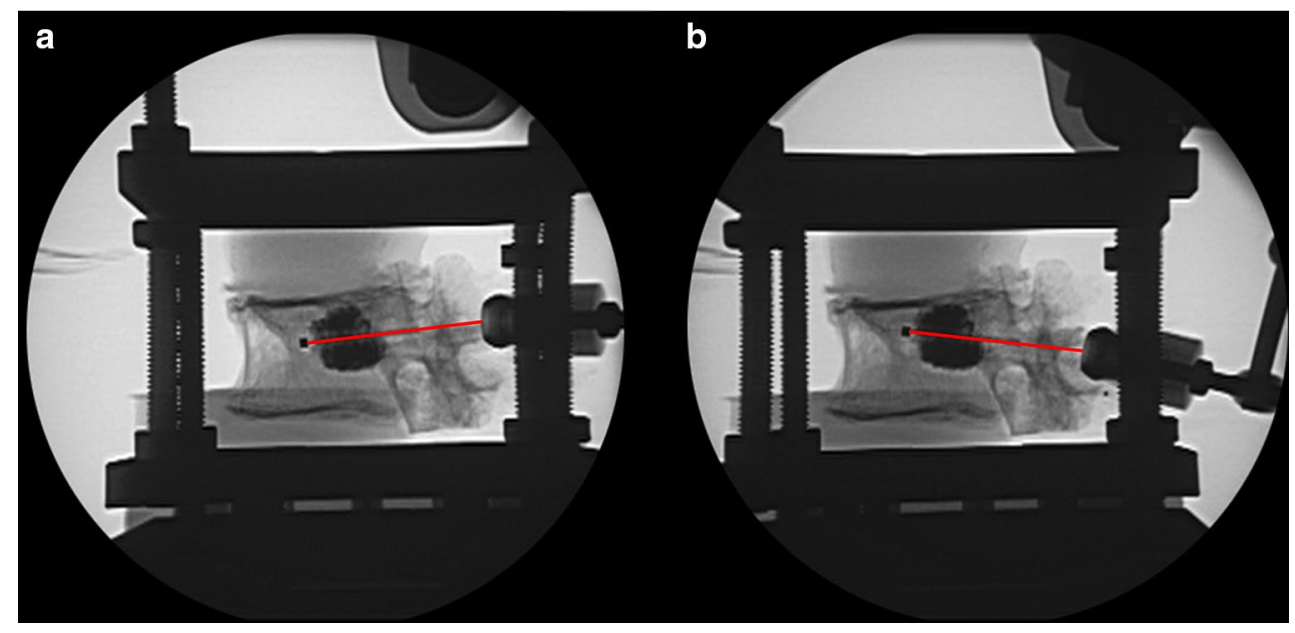

Fig. 3 Exemplary stress fluoroscopy of a PMMA-augmented CF/PEEK pedicle screw after loosening. Lateral fluoroscopic images were taken under minimal (tension) (a) and maximal (compression) (b) loads. Screw axis is marked in red 


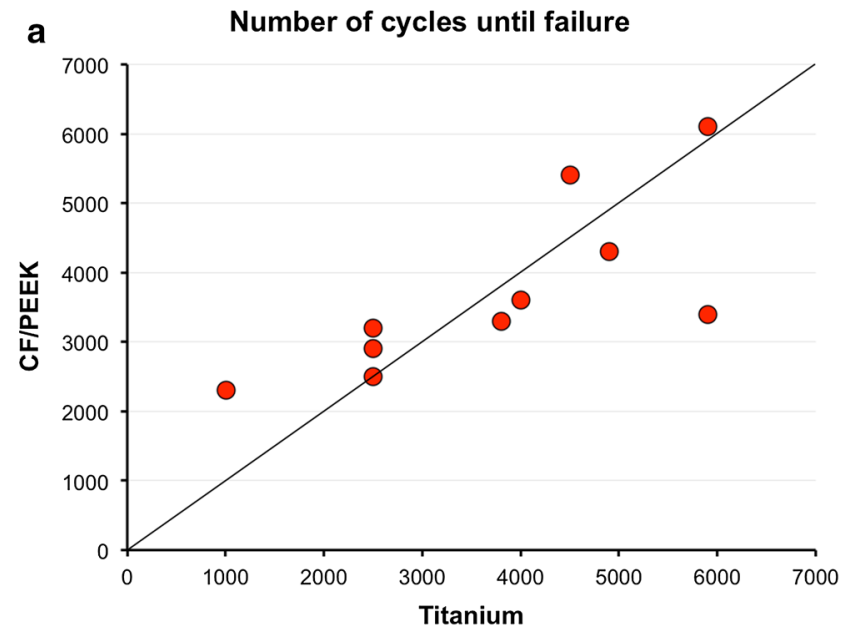

Fig. 4 Scatter plots of number of cycles until failure. One dot represents two screws of one vertebra. a CF/PEEK vs. standard titanium pedicle screws randomly placed into either the left or right pedicle of

$\mathrm{CF} / \mathrm{PEEK}$ and titanium pedicle screws $\left[0.5^{\circ} \pm 0.6^{\circ}\right.$ vs. $0.7^{\circ} \pm 0.8^{\circ}(p=0.12) ; 3.7^{\circ} \pm 1.6^{\circ}$ vs. $3.3^{\circ} \pm 2.1^{\circ}(p=0.72)$; and $14.5^{\circ} \pm 3.1^{\circ}$ vs. $14.9^{\circ} \pm 2.8^{\circ}(p=0.73)$, respectively].

\section{PMMA-augmented vs. nonaugmented CF/PEEK pedicle screws}

For comparison of PMMA-augmented vs. nonaugmented CF/PEEK pedicle screws, CF/PEEK pedicle screws were placed into the left and right pedicle of each of ten isolated human vertebrae [BMD $56.3 \pm 15.8 \mathrm{mg} / \mathrm{cm}^{3}$ (range 37.1-82.0)]. One screw of each vertebra was randomly selected and augmented with $2 \mathrm{ml}$ PMMA cement. All screws loosened within the 10,000 load cycles.

PMMA cement augmentation of CF/PEEK pedicle screws significantly increased the number of load cycles until loosening by 1.63 -fold [ $5100 \pm 1933$ (corresponding to $305 \pm 97 \mathrm{~N}$ ) in augmented vs. $3130 \pm 2132$ load cycles (corresponding to $206.5 \pm 107 \mathrm{~N}$ ) in nonaugmented $\mathrm{CF} /$ PEEK screws, $p=0.015]$. In 8 out of 10 vertebrae, the PMMA-augmented screws reached a higher number of load cycles until loosening than the contralateral nonaugmented screws (Fig. 4). Correlation between number of load cycles until loosening and BMD was very weak in augmented $(r=-0.18, p=0.61)$ and moderate in nonaugmented screws $(r=0.56, p=0.09)$.

Angular screw motion assessed by stress fluoroscopy did not significantly differ at 1000 and 2000 cycles, but was significantly smaller in augmented than in nonaugmented $\mathrm{CF} / \mathrm{PEEK}$ screws at last measurement before $\left(2.3^{\circ} \pm 1.1^{\circ} \mathrm{vs}\right.$. $\left.4.2^{\circ} \pm 1.3^{\circ} ; p=0.009\right)$ as well as at first measurement after failure $\left(10.5^{\circ} \pm 3.2^{\circ}\right.$ vs. $\left.17.1^{\circ} \pm 2.9^{\circ} ; p=0.022\right)$.

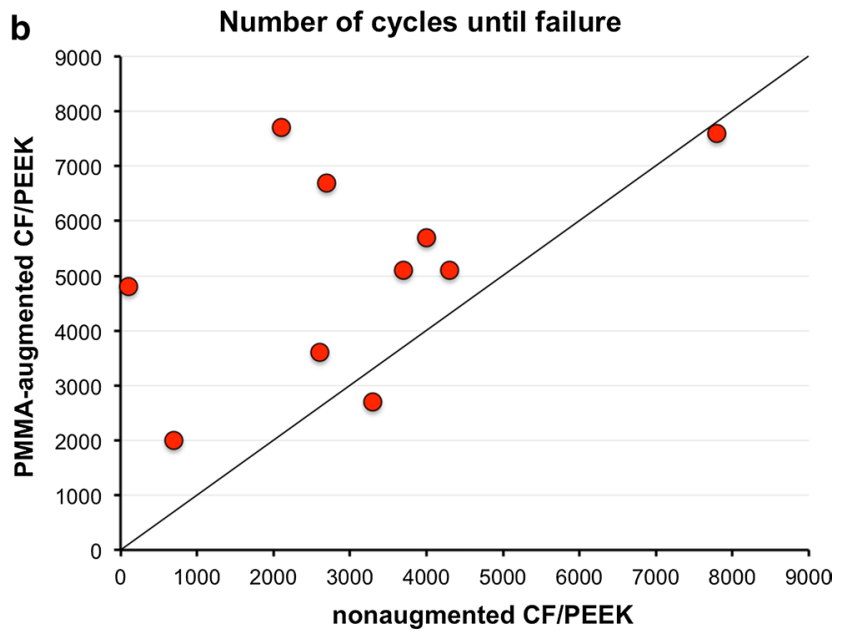

the same vertebra. b PMMA-augmented vs. nonaugmented CF/PEEK pedicle screws randomly placed into either the left or right pedicle of the same vertebra

\section{Discussion}

The results from this biomechanical study show that (1) the nonmetallic CF/PEEK pedicle screws resisted a similar number of load cycles until loosening as standard titanium screws and that (2) PMMA cement augmentation of CF/ PEEK screws significantly increases the number of load cycles until loosening.

To the best of our knowledge this is the first study that biomechanically evaluates CF/PEEK pedicle screws. $\mathrm{CF} /$ PEEK pedicle screws have only recently become available, and PEEK and reinforced PEEK composites as a material for screw/rod constructs have been suggested to hold the potential to overcome major drawbacks of standard titanium. PEEK is a radiolucent, biocompatible thermoplastic polymer with greater strength per mass than many metals and suitable for load-bearing orthopaedic implants. CF/PEEK is even superior to neat PEEK in terms of wear resistance and mechanical properties [3]. CF/PEEK with its unique mechanical properties may thus represent a desirable compromise between stiff titanium and distinctly more flexible neat PEEK, and consequently may provide optimal stiffness to act as an adjunct to fusion. Several previous studies [22] biomechanically evaluated neat PEEK and CF/PEEK rods for posterolateral fusion, posterior lumbar interbody fusion and anterior column reconstruction constructs. PEEK and CF/PEEK rods were found to provide comparable biomechanical stability and restriction of range of motion to titanium rods [20,27, 28]. Due to the lower modulus of elasticity, PEEK rods furthermore provided a more physiological anterior column load-sharing profile [18, 29, 30]. The increased anterior column load-sharing again appears to reduce stresses at the bone-screw interface $[17,18]$ and 
may promote interbody fusion [20,31], particularly when the interbody graft is slightly undersized or partially subsided. Moreover, lower stresses, intradiscal pressures and loss of disc height have been found at the cranial adjacent level in PEEK compared to titanium rod constructs [17, 32], suggesting that PEEK rods might be beneficial to lessen the probability of adjacent level disease. In contrast to these previous studies on PEEK rods, the present study evaluated the biomechanical performance of CF/PEEK pedicle screws and specifically focused on pedicle screw loosening under cyclic cranio-caudal loading.

The etiology of pedicle screw loosening is still insufficiently understood but contributing factors include poor bone quality, stress shielding effects of rigid metallic screw/ rod constructs, insufficient anterior support, and possibly wear debris-induced osteolysis and implant-related infection [7]. PEEK and CF/PEEK screw/rod constructs for posterior instrumentation may hold significant advantages over titanium constructs with regard to screw loosening. First, rigid metallic screw/rod constructs shift the physiological loads posteriorly and unload the anterior column (stress shielding) resulting in increased stresses at the anchoring points of the construct (i.e., the bone-screw interface) [20]. In contrast, PEEK and CF/PEEK screw/rod constructs allow for increased anterior column load-sharing due to the lower elastic modulus, and thus more closely mimic physiologic loading $[18,29,30]$. Increased anterior column load-sharing reduces stresses at the bone-screw interface $[17,18]$ and consequently may decrease the risk of screw loosening until bony fusion occurs. Second, the elastic modulus of PEEK and CF/PEEK pedicle screws matches that of the surrounding bone more closely compared to metallic screws, which may additionally decrease the risk of screw loosening. The reduced modulus mismatch between screw and bone theoretically results in a more homogeneous stress distribution and less micromotion due to similar strains of the screw material and the surrounding bone, particularly in osteoporotic bone, and may lead to enhanced osseointegration and improved screw fixation strength [19, 33, 34].

In accordance with our first hypothesis, CF/PEEK and titanium pedicle screws resisted a similar number of load cycles until loosening. Despite the lower elastic modulus, however, CF/PEEK screws were not superior in terms of loosening. A possible explanation for the latter finding may be that the reduced modulus mismatch between screw and bone affects "primary stability" and resistance to early screw loosening only to a lesser extent, but rather improves longterm screw fixation strength via osseointegration and bone remodelling around the screws. In an elaborate study, Shi et al. [19] were able to show that axial pull-out strength and peri-implant bone formation is significantly enhanced in expandable pedicle screws of low elastic modulus (Ti-24Nb4Zr-7.9Sn, $42 \mathrm{GPa}$ ) as compared to standard titanium screws
(Ti-6Al-4 V, $110 \mathrm{GPa}) 6$ months after implantation in osteoporotic sheep. Their finding that implant modulus affects peri-implant bone remodelling is in accordance with previous reports [33,34]. At an early stage after screw placement, however, bone quality and BMD might be the main determining factor of screw fixation strength and risk of loosening [35]. A potential clinically relevant effect of the modulus differences between titanium and CF/PEEK screws therefore may not come to light in biomechanical testing which evidently cannot take into account osseointegration and bone remodelling around the screws. In vivo animal implantation studies and clinical studies thus will be needed to clarify whether low elastic modulus CF/PEEK pedicle screws are advantageous over conventional high elastic modulus titanium screws as to long-term screw loosening. To the best of our knowledge, there are only two preliminary clinical studies reporting on first experiences with CF/PEEK pedicle screws in a total of 44 patients (thirty-nine with spinal tumors and five with degenerative disease) $[4,5]$. These studies conclude that implantation of CF/PEEK implants was feasible and that postoperative imaging and dose calculation for radiotherapy was facilitated due to reduced artifacts. These preliminary reports, however, cannot answer the question whether pedicle screw anchorage of CF/PEEK screws is comparable to that of standard titanium screws.

As expected, PMMA cement augmentation significantly enhanced screw anchorage of CF/PEEK screws. This finding is well in line with observations from previous studies evaluating the effect of cement augmentation in metallic pedicle screws [14]. In contrast to Weiser et al. [35], we only found a weak to moderate correlation (with Spearman's correlation coefficients ranging from 0.33 to 0.56 ) between BMD and number of cycles until loosening for both nonaugmented CF/PEEK and titanium screws. The poor correlation between BMD and number of cycles until loosening may be explained by the small range in BMD of specimens tested as well as by the small sample size. Moreover, pedicle width and length were not determined in the present study and may also influence screw anchorage. When combining all nonaugmented CF/PEEK and titanium screws $(n=30)$, correlation between BMD and number of cycles until loosening was still moderate (Spearman's correlation coefficient: 0.45 ) but reached significance $(p=0.013)$. In augmented CF/ PEEK screws, this correlation was even weaker $(r=-0.18$, $p=0.61$, which may be a consequence of an interlocking between the cement and the cancellous bone around the screws. The latter probably outweighs the minor differences in BMD among specimens tested [36].

Various research groups developed different methods to investigate pedicle screw anchorage with more elaborate test setups than simple non-physiological pull-out tests. They all applied cyclic loading with an increasing load magnitude to provoke screw loosening. In addition 
to the compressive load, the present setup also includes a tensional load component and was thereby capable of reproducing the "windscreen wiper motion" of the screw which is usually seen in in vivo screw loosening. Similar to the present test setup, Kueny et al. [37] and Bostelmann et al. [25] used a toggle test setup which allowed translation of the vertebra in the transverse plane via an $x-y$ table during loading. While Kueny et al. [37] introduced the load on the screw head, resulting in an axial loading superimposed by a bending moment; the present test setup applied the load via a rod and an offset rotational axis. The latter results in an axial loading superimposed by a bending moment with an additional shear component. The shear component, however, is neutralized by the translation of the $x-y$ table. In contrast to these single pedicle screw loading tests allowing for pairwise left-right comparisons under comparable conditions, Wilke et al. [38] used entire assemblies of bisegmentally instrumented spinal motion segments. While this test setup tests the whole clinically applied instrumentation, it does not allow for control of numerous confounding factors related to specimen variation (e.g., BMD, vertebrae morphology, pedicle morphology). Due to multiple confounding factors smaller differences between test groups may be difficult to detect and require an unreasonable high number of test samples. Yet this setup enables a more comprehensive multidirectional loading involving a combination of axial loads and bending moments varying from flexion/extension to lateral bending as well as combinations of those. Recently, Liebsch et al. [39] reported a test setup which also allows for a pairwise left-right comparison of pedicle screw anchorage. They proposed an elaborate loading with an axial load superimposed by a bending moment as well as by a shear force in direction of the screw axis. The axial load was introduced physiologically through the vertebral body with the construct fixed to the machine base by a rod. This test setup enables a multicomponent loading of the pedicle screw with combined axial and shear forces and a bending moment. However, with adding a shear force component, an additional constraint to restrict the translation of the screw in direction of the screw axis is introduced. By introducing the load through the vertebra, the peak bending moment is more physiologically located at the screwrod connection and not at the screw entry point in the vertebra as in toggle tests. In this setup, however, screw anchorage might be very sensitive to the exact position of load application in relation to the screw tip position. While this can be well controlled with standardized synthetic bone surrogates, it may pose a challenge to control this parameter in cadaver tests with left-right comparisons and/or with specimens of varying anatomy. To summarize, all of these test setups have been reported to reproduce an in vivo like screw loosening pattern and all test setups described in the literature so far have their advantages and disadvantages. While a toggle test setup simulates axial loading superimposed by a bending moment without constraints, it introduces the load in the screw head. The multicomponent loading test setup represents a more constraint system, however, it allows to also simulate shear force components and applies the load more physiologically in the vertebra.

A limitation of this study is that biomechanical testing of screws implanted into cadaveric vertebrae cannot take into account implant osseointegration, which determines medium- and long-term fixation strength of loadbearing implants. Moreover, despite our elaborate cyclic cranio-caudal loading protocol, biomechanical testing of cadaveric specimens cannot fully reflect but rather only approximate in vivo loading conditions. As screw loosening most commonly occurs in osteoporotic bone, the tested specimens were obtained from elderly individuals with low BMD; extrapolation of our findings to high-density bone, however, should be treated with caution. Finally, our study did not include a comparison between PMMAaugmented CF/PEEK and PMMA-augmented titanium screws as the number of available cadaveric specimens was limited. Such a comparison could add further valuable information. However, as screw anchorage of nonaugmented $\mathrm{CF} / \mathrm{PEEK}$ and nonaugmented titanium screws did not significantly differ, it may be reasonable to assume that screw anchorage of PMMA-augmented CF/PEEK and titanium screws is comparable as well.

There are several strengths of this study. First, to the best of our knowledge, this is the first study evaluating the biomechanical performance of CF/PEEK pedicle screws. Previous studies only investigated PEEK and CF/PEEK rods connected to titanium pedicle screws. However, as also noted by Bruner et al. [27], evaluation of screw/rod constructs entirely made of PEEK and CF/PEEK is essential to improve our understanding of potential advantages these materials may offer for spinal instrumentation. Second, the elaborate load protocol of this study included cyclic cranio-caudal loading with stepwise increasing loads until screw loosening or a maximum of 10,000 load cycles. Cyclic cranio-caudal loading is considered more appropriate to assess and simulate in vivo screw loosening than much more commonly used and easier performed pull-out tests $[14,25,40]$. Third, CF/PEEK and titanium control screws were randomly placed into either the left or right pedicle of the same vertebra, and screws were of different material, but of identical design. This experimental design thus allowed for a standardized pairwise comparison and for controlling for potential confounding factors, such as specimen variation (particularly in bone quality and pedicle morphology). 


\section{Conclusions}

Nonmetallic CF/PEEK pedicle screws resisted a similar number of load cycles until loosening as standard titanium screws. In addition, PMMA cement augmentation of $\mathrm{CF} /$ PEEK screws significantly increased the number of load cycles until loosening by 1.63 -fold. The presented biomechanical test setup involved stepwise increasing cyclic cranio-caudal loading until pedicle screw loosening occurred and used cadaveric specimens with low BMD as highest loosening rates have been reported for osteoporotic individuals. Nevertheless, further in vivo studies are needed to determine whether long-term screw anchorage via osseointegration is enhanced in CF/PEEK as compared to standard titanium screws. While comparable in terms of loosening under cyclic loads, the nonmetallic screws hold clinically significant advantages over the conventional metallic ones. Unlike titanium screws, radiolucent CF/PEEK screws facilitate postoperative imaging and do not interfere with radiotherapy. CF/PEEK therefore may offer a valuable nonmetallic alternative to standard titanium for screw/rod constructs.

Acknowledgements Open access funding provided by University of Innsbruck and Medical University of Innsbruck. The authors would like to thank Daniela Kramer and Tatjana Schneeweis for technical assistance, and express their gratitude to individuals who donated their bodies and tissues for the advancement of education and research.

\section{Compliance with ethical standards}

Conflict of interest Laboratory costs were supported by icotec ag, Altstätten, Switzerland. T. N. is employed by icotec ag, Altstätten, Switzerland. None of the remaining authors has a conflict of interest and none received payment of any sort for their contributions to this work. The funding sources had no involvement in the study design; in the collection, analysis and interpretation of data; or in the decision to submit the paper for publication.

Open Access This article is distributed under the terms of the Creative Commons Attribution 4.0 International License (http://creativeco mmons.org/licenses/by/4.0/), which permits unrestricted use, distribution, and reproduction in any medium, provided you give appropriate credit to the original author(s) and the source, provide a link to the Creative Commons license, and indicate if changes were made.

\section{References}

1. Stradiotti P, Curti A, Castellazzi G, Zerbi A (2009) Metal-related artifacts in instrumented spine. Techniques for reducing artifacts in CT and MRI: state of the art. Eur Spine J 18(Suppl 1):102-108

2. Rudisch A, Kremser C, Peer S, Kathrein A, Judmaier W, Daniaux $\mathrm{H}$ (1998) Metallic artifacts in magnetic resonance imaging of patients with spinal fusion-a comparison of implant materials and imaging sequences. Spine 23:692-699

3. Kurtz SM, Devine JN (2007) PEEK biomaterials in trauma, orthopedic, and spinal implants. Biomaterials 28:4845-4869
4. Eicker SO, Krajewski K, Payer S, Krätzig T, Dreimann M (2017) First experience with carbon/PEEK pedicle screws. J Neurosurg Sci 61:222-224

5. Ringel F, Ryang YM, Kirschke JS, Müller BS, Wilkens JJ, Brodard J, Combs SE, Meyer B (2017) Radiolucent carbon fiber-reinforced pedicle screws for treatment of spinal tumors: advantages for radiation planning and follow-up imaging. World Neurosurg 105:294-301

6. Nevelsky A, Borzov E, Daniel S, Bar-Deroma R (2017) Perturbation effects of the carbon fiber-PEEK screws on radiotherapy dose distribution. J Appl Clin Med Phys 18:62-68

7. Galbusera F, Volkheimer D, Reitmaier S, Berger-Roscher N, Kienle A, Wilke HJ (2015) Pedicle screw loosening: a clinically relevant complication? Eur Spine J 24:1005-1016

8. Boos N, Webb JK (1997) Pedicle screw fixation in spinal disorders: a European view. Eur Spine J 6:2-18

9. Röllinghoff M, Schlüter-Brust K, Groos D, Sobottke R, Michael JW, Eysel P, Delank KS (2010) Mid-range outcomes in 64 consecutive cases of multilevel fusion for degenerative diseases of the lumbar spine. Orthop Rev 2:e3

10. Bredow J, Boese CK, Werner CML, Siewe J, Löhrer L, Zarghooni K, Eysel P, Scheyerer MJ (2016) Predictive validity of preoperative CT scans and the risk of pedicle screw loosening in spinal surgery. Arch Orthop Trauma Surg 136:1063-1067

11. El Saman A, Meier S, Sander A, Kelm A, Marzi I, Laurer H (2013) Reduced loosening rate and loss of correction following posterior stabilization with or without PMMA augmentation of pedicle screws in vertebral fractures in the elderly. Eur J Trauma Emerg Surg 39:455-460

12. Pearson HB, Dobbs CJ, Grantham E, Niebur GL, Chappuis JL, Boerckel JD (2017) Intraoperative biomechanics of lumbar pedicle screw loosening following successful arthrodesis. J Orthop Res 35:2673-2681

13. Shea TM, Laun J, Gonzalez-Blohm SA, Doulgeris JJ, Lee WE 3rd, Aghayev K, Vrionis FD (2014) Designs and techniques that improve the pullout strength of pedicle screws in osteoporotic vertebrae: current status. Biomed Res Int 2014:748393

14. Elder BD, Lo SF, Holmes C, Goodwin CR, Kosztowski TA, Lina IA, Locke JE, Witham TF (2015) The biomechanics of pedicle screw augmentation with cement. Spine J 15:1432-1445

15. Ohtori S, Inoue G, Orita S, Yamauchi K, Eguchi Y, Ochiai N, Kishida S, Kuniyoshi K, Aoki Y, Nakamura J, Ishikawa T, Miyagi M, Kamoda H, Suzuki M, Kubota G, Sakuma Y, Oikawa Y, Inage K, Sainoh T, Takaso M, Toyone T, Takahashi K (2013) Comparison of teriparatide and bisphosphonate treatment to reduce pedicle screw loosening after lumbar spinal fusion surgery in postmenopausal women with osteoporosis from a bone quality perspective. Spine 38:E487-E492

16. Mavrogenis AF, Vottis C, Triantafyllopoulos G, Papagelopoulos PJ, Pneumaticos SG (2014) PEEK rod systems for the spine. Eur J Orthop Surg Traumatol 24(Suppl 1):S111-S116

17. Chou WK, Chien A, Wang JL (2015) Biomechanical analysis between PEEK and titanium screw-rods spinal construct subjected to fatigue loading. J Spinal Disord Tech 28:E121-E125

18. Turner JL, Paller DJ, Murrell CB (2010) The mechanical effect of commercially pure titanium and polyetheretherketone rods on spinal implants at the operative and adjacent levels. Spine 35:E1076-E1082

19. Shi L, Wang L, Guo Z, Wu ZX, Liu D, Gao MX, Wan SY, Fu SC, Li SJ, Lei W (2012) A study of low elastic modulus expandable pedicle screws in osteoporotic sheep. J Spinal Disord Tech 25:123-128

20. Ponnappan RK, Serhan H, Zarda B, Patel R, Albert T, Vaccaro AR (2009) Biomechanical evaluation and comparison of polyetheretherketone rod system to traditional titanium rod fixation. Spine J 9:263-267 
21. Panayotov IV, Orti V, Cuisinier F, Yachouh J (2016) Polyetheretherketone (PEEK) for medical applications. J Mater Sci Mater Med 27:118

22. Li C, Liu L, Shi JY, Yan KZ, Shen WZ, Yang ZR (2016) Clinical and biomechanical researches of polyetheretherketone (PEEK) rods for semi-rigid lumbar fusion: a systematic review. Neurosurg Rev. https://doi.org/10.1007/s10143-016-0763-2

23. Kersten RFMR, van Gaalen SM, de Gast A, Öner FC (2015) Polyetheretherketone (PEEK) cages in cervical applications: a systematic review. Spine J 15:1446-1460

24. Li CS, Vannabouathong C, Sprague S, Bhandari M (2015) The use of carbon-fiber-reinforced (CFR) PEEK material in orthopedic implants: a systematic review. Clin Med Insights Arthritis Musculoskelet Disord 8:33-45

25. Bostelmann R, Keiler A, Steiger HJ, Scholz A, Cornelius JF, Schmoelz W (2017) Effect of augmentation techniques on the failure of pedicle screws under cranio-caudal cyclic loading. Eur Spine J 26:181-188

26. Schmoelz W, Heinrichs CH, Schmidt S, Piñera AR, TomeBermejo F, Duart JM, Bauer M, Galovich LÁ (2017) Timing of PMMA cement application for pedicle screw augmentation affects screw anchorage. Eur Spine J 26:2883-2890

27. Bruner HJ, Guan Y, Yoganandan N, Pintar FA, Maiman DJ, Slivka MA (2010) Biomechanics of polyaryletherketone rod composites and titanium rods for posterior lumbosacral instrumentation. Presented at the 2010 Joint Spine Section Meeting. Laboratory investigation. J Neurosurg Spine 13:766-772

28. Yeager MS, Cook DJ, Cheng BC (2015) In vitro comparison of Dynesys, PEEK, and titanium constructs in the lumbar spine. Adv Orthop 2015:895931

29. Ahn YH, Chen WM, Lee KY, Park KW, Lee SJ (2008) Comparison of the load-sharing characteristics between pedicle-based dynamic and rigid rod devices. Biomed Mater 3:044101

30. Zhou R, Huang Z, Liu X, Tong J, Ji W, Liu S, Zhu Q (2016) Kinematics and load-sharing of an anterior thoracolumbar spinal reconstruction construct with PEEK rods: an in vitro biomechanical study. Clin Biomech (Bristol, Avon) 40:1-7

31. Wang N, Xie H, Xi C, Zhang H, Yan J (2017) A study to compare the efficacy of polyether ether ketone rod device with titanium devices in posterior spinal fusion in a canine model. J Orthop Surg Res 12:40

32. Jin YJ, Kim YE, Seo JH, Choi HW, Jahng TA (2013) Effects of rod stiffness and fusion mass on the adjacent segments after floating mono-segmental fusion: a study using finite element analysis. Eur Spine J 22:1066-1077

33. Lin DJ, Chuang CC, Chern Lin JH, Lee JW, Ju CP, Yin HS (2007) Bone formation at the surface of low modulus Ti-7.5Mo implants in rabbit femur. Biomaterials 28:2582-2589

34. Stoppie N, Van Oosterwyck H, Jansen J, Wolke J, Wevers M, Naert I (2009) The influence of Young's modulus of loaded implants on bone remodeling: an experimental and numerical study in the goat knee. J Biomed Mater Res 90A:792-803

35. Weiser L, Huber G, Sellenschloh K, Viezens L, Püschel K, Morlock MM, Lehmann W (2017) Insufficient stability of pedicle screws in osteoporotic vertebrae: biomechanical correlation of bone mineral density and pedicle screw fixation strength. Eur Spine J 26:2891-2897

36. Hoppe S, Loosli Y, Baumgartner D, Heini P, Benneker L (2014) Influence of screw augmentation in posterior dynamic and rigid stabilization systems in osteoporotic lumbar vertebrae: a biomechanical cadaveric study. Spine 39:E384-E389

37. Kueny RA, Kolb JP, Lehmann W, Püschel K, Morlock MM, Huber $\mathrm{G}$ (2014) Influence of the screw augmentation technique and a diameter increase on pedicle screw fixation in the osteoporotic spine: pullout versus fatigue testing. Eur Spine J 23:2196-2202

38. Wilke HJ, Kaiser D, Volkheimer D, Hackenbroch C, Püschel K, Rauschmann M (2016) A pedicle screw system and a lamina hook system provide similar primary and long-term stability: a biomechanical in vitro study with quasi-static and dynamic loading conditions. Eur Spine J 25:2919-2928

39. Liebsch C, Zimmermann J, Graf N, Schilling C, Wilke HJ, Kienle A (2018) In vitro validation of a novel mechanical model for testing the anchorage capacity of pedicle screws using physiological load application. J Mech Behav Biomed Mater 77:578-585

40. Rohlmann A, Bergmann G, Graichen F (1999) Loads on internal spinal fixators measured in different body positions. Eur Spine $\mathbf{J}$ $8: 354-359$ 